This is the final peer-reviewed accepted manuscript of:

R. Veisllari, S. Bjornstad, J. P. Braute, K. Bozorgebrahimi and C. Raffaelli, "Field-trial demonstration of cost efficient sub-wavelength service through integrated packet/circuit hybrid network [invited]," in Journal of Optical Communications and Networking, vol. 7, no. 3, pp. A379-A387, March 2015.

The final published version is available online at:

https://doi.org/10.1364/JOCN.7.00A379

Rights / License:

The terms and conditions for the reuse of this version of the manuscript are specified in the publishing policy. For all terms of use and more information see the publisher's website.

This item was downloaded from IRIS Università di Bologna (https://cris.unibo.it/)

When citing, please refer to the published version. 


\title{
Field-Trial Demonstration of Cost Efficient Sub-Wavelength Service through Integrated Packet/Circuit Hybrid Network [Invited]
}

\author{
Raimena Veisllari, Steinar Bjornstad, Jan P. Braute, Kurosh Bozorgebrahimi, \\ and Carla Raffaelli
}

\begin{abstract}
Carriers are under a constant pressure to meet the ever-increasing bandwidth demand while reducing cost per bit, enhancing network throughput and offering a large variety of service. Hybrid packet and circuit network technologies are being widely investigated and considered as a solution for offering both the high network throughput of the packet domain and wavelength services, i.e. a low fixed latency and zero packet loss. To enable carriers to serve a higher number of customers requiring wavelength-services, OTN-based sub-wavelength switching is adopted to support finer granularity with similar performance to full wavelength services. However, OTN is not able to do statistical multiplexing and achieve the throughput efficiency of packet networks. In this work an integrated hybrid optical network field-trial is described to demonstrate the ability to both aggregate and transport sub-wavelength circuits, and offer high throughput efficiency by statistical multiplexing traffic on transport wavelengths. Results show the transport of sub-wavelength services with packet delay variation limited to only $15 \mathrm{~ns}$ and $82.4 \%$ wavelength utilization using statistical multiplexing.
\end{abstract}

Index Terms - circuit-switched; field-trial; packetswitched; hybrid networks; sub-wavelength.

\section{INTRODUCTION}

Carriers struggle to keep pace with the ever increasing

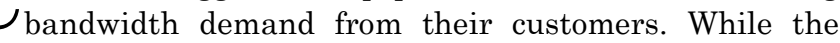

Manuscript received July 1, 2014.

R. Veisllari and S. Bjornstad are with the Dept. of Telematics, NTNU, Trondheim 7041, Norway, \{veisllar; steinar.bjornstad\} @item.ntnu.no.

S. Bjornstad is also with TransPacket AS, Oslo 0277, Norway.

J. P. Braute is with TransPacket AS, Oslo 0277, Norway.

$\mathrm{K}$. Bozorgebrahimi is with the UNINETT AS, Trondheim 7465 , Norway.

C. Raffaelli is with DEI, University of Bologna, viale Risorgimento 2, 40136, Bologna, Italy. demand for bandwidth is constantly increasing, there is not a strong willingness to pay for the additional bandwidth. This motivates for systems and mechanisms that can increase the bandwidth utilization of the network without adding significant costs. The utilization of wavelengths in optical networks is in many cases low due to e.g. a temporarily low traffic load or bandwidth reserved for resiliency purposes $[1,2]$. With the ever increasing channel bitrates in optical communication systems now targeting $\mathrm{Tb} / \mathrm{s}$ capacities, the need for splitting the bandwidth into sub-wavelength capacities with the characteristics of wavelengths for aggregation purposes becomes increasingly important. This allows carriers to offer wavelength type of services to their customers with a lower bitrate than the physical wavelength, e.g. by aggregating $1 \mathrm{~Gb} / \mathrm{s}$ customer links into a $10 \mathrm{~Gb} / \mathrm{s}$ physical transport wavelength.

Optical Transport Network (OTN) [3-5] offers a circuit switched approach to aggregation and switching of subwavelengths, allowing zero packet loss, low-latency, ultralow packet-delay variation and synchronization transport. These characteristics enable support for a variety of applications and systems needing timing-critical transport. Strict timing is a key requirement for e.g. mobile backhaul networks, as well as for wavelength-services and private lines in sub-wavelengths [6, 7]. Sub-wavelengths allow full isolation between the services on both throughput and timing. Packet networks on the other hand, offer a higher throughput by using statistical multiplexing, but do not match OTN network timing-performance. Hence, there is currently a wide consensus in the optical networking research community and service providers regarding the need for fully integrated systems that combine the properties of circuit switched, e.g. OTN, and packet switched networks into a single network [8, 9]. Many packet-optical transport architectures employ packet, OTN and WDM switching in the same node [10, 11]. However, the OTN sub-rate assigned to a client cannot be statistically multiplexed with traffic from other clients and is therefore not re-used like in packet networks. This prevents optimization of resource utilization at the sub-wavelength layer.

Integrated hybrid optical networks (IHON) [12-19], merge the circuit and packet network in the same wavelength to 
enable: (1) circuit quality transport of demanding services; (2) circuits with higher granularity than wavelengths, i.e. sub-wavelength switching; (3) statistical multiplexing enabling the throughput efficiency of packet networks. Earlier IHON experiments have demonstrated how the timing is preserved on a single circuit class stream, referred to as Guaranteed Service Transport (GST), while adding Statistically Multiplexed (SM) packet-switched traffic [16].

In this work is proposed an enhancement of the Fusion implementation of the integrated hybrid optical network with the ability to transport wavelength services at subwavelength granularity [17]. The novel aggregation scheme enables several lower bitrate GST packet streams, i.e. subwavelengths, to be aggregated into, and de-aggregated from, a higher bitrate GST packet stream following a wavelength. We demonstrate experimentally that GST enables subwavelengths with circuit-switched quality of service (QoS), i.e., absolute transfer guarantees with no packet loss, low packet delay and ultra-low packet delay variation (PDV). For maintaining the circuit QoS, i.e. avoiding PDV, GST preserves both packet-lengths and packet gaps when being aggregated into a higher bitrate stream. This enables accurate reconstruction of the stream at the destination. Any capacity not utilized by the GST packets is identified in the packet stream as idle time-gaps. The lightpath utilization is increased by filling these gaps with packets through statistical multiplexing of packets from the lower priority SM class. In addition, the channel utilization and SM traffic throughput is further characterized by simulation and two analytical models are adapted to quantify the maximum achievable throughput. The found threshold is applicable in the network designing process to optimize the system dimension.

The outline of the paper is as follows. Section II introduces the integrated hybrid optical networks and related work. The Fusion node, an implementation of the IHON architectures, employed in the field-trial and the subwavelength aggregation scheme are described in Section III. In Section IV is presented the field-trial setup and related results. The simulation results and analysis on the wavelength utilization are offered in Section V. In Section VI is discussed on IHON and its Fusion implementation and the applicability in the optical network. We draw our conclusions in Section VII.

\section{INTEGRATED HYBRID OPTICAL NETWORKS}

Integrated hybrid optical networks, also known as integrated packet/circuit networks, have the distinctive characteristic of transmitting two main traffic classes, namely circuit and packet, on the same physical resource, i.e. wavelength. The wavelengths are provisioned exclusively for the circuit traffic. Hence, this traffic is transported with strict guaranteed quality of service: no packet jitter, low deterministic delay comparable to the propagation delay and no loss. Provisioning circuits of wavelength granularity leads to the well-known issue of low resource utilization in optical circuit switching and wavelength routed optical networks (WRON). To maximize throughput, IHON detects the idle time-gaps in between the packets of the circuit stream and inserts packets from the best effort traffic class if they fit in the gaps. Thus, the circuit traffic is oblivious to- and not affected by- the additional packet traffic. We refer to the traffic class transported in the circuit as guaranteed service transport (GST) and to the low priority added packet traffic as statistically multiplexed (SM).

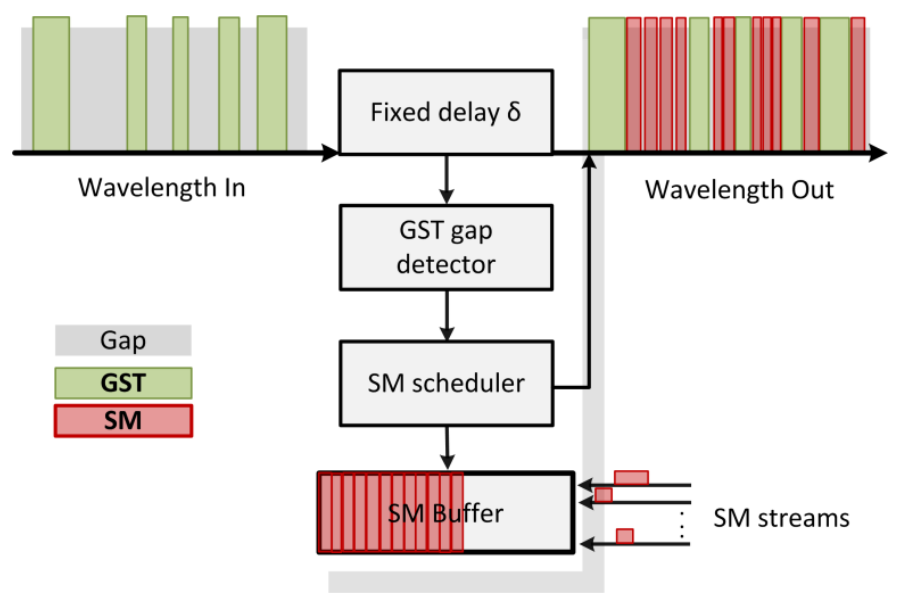

Fig. 1. The GST packets are delayed for a fixed time, corresponding to the SM maximum transmission unit (MTU), before accessing the output channel. This delay enables the GST gap detector to detect the GST in the input and update the information on the free gaps before SM insertion. The SM scheduler looks for packets at the head of the SM queues and schedules them only if they fit in the gaps, to avoid SM preemption.

Figure 1 illustrates the main building blocks of the IHON node for SM insertion at an output wavelength provisioned for GST. At each node, before accessing the output channel, the GST traffic passes through a fixed delay $\delta$ corresponding to the transmission time in the channel of capacity $C$ of a maximum length $\mathrm{SM}$ packet $L_{\max }$, i.e. $\delta=L_{\max } / C$, and prevents preemption of SM by incoming GST packets. For example, if the channel is free and no GST is detected in the delay line at time $t_{0}$, the SM scheduler will start the transmission of an SM packet of size $L \leq L_{\max }$. In case that a GST packet is detected immediately after that at the input of the delay line at time $t_{1}>t_{0}$, the delay ensures that the GST arrival at the output channel does not preempt the SM packet as the arrival time is after SM has finished service, i.e. $\left(t_{1}+L_{\max } / C\right)>\left(t_{0}+L / C\right)$. The effect of $\delta$ on the end-to-end GST delay is deterministic and in addition it allows the GST gap detector to detect the duration of idle time-gaps between GST packets in the channel. This information is used by the SM scheduler checking the SM input queue(s) for a packet of suitable size that fits the gap. If a suitable packet is found, the SM packet is inserted without affecting the timing of the packets in the GST stream, while increasing the throughput in the wavelength.

\section{A. Related work}

The integrated hybrid node has been well investigated in literature with proposed architectures from all-optical [14, $15,19]$ to hybrid electronic-optical $[13,18]$ and all-electronic [16]. The main difference of the integrated with the parallel hybrid optical network (PHON), as defined first in [12], is that while in the parallel hybrid the packet and circuit 
domains might share the same physical fiber but have different separate wavelength pools, in IHON the packet domain, i.e. SM, is able to use the physical wavelengths provisioned for the circuit domain, i.e. for GST.

Recent work on PHON architectures has increased the flexibility of re-assigning the wavelengths in a dynamic manner as to adapt to the traffic demand from each class [20]. Nevertheless, at a fixed point in time, the wavelength is always belonging to only a single specific class: either circuit or packet. Thus, the circuit wavelengths might still be under-utilized. The integrated hybrid goes one step further and enables the use of these wavelengths, currently provisioned for circuits, by the lower priority packet traffic. The latter can even be further differentiated into multiple priority classes, e.g. in [14] a real-time SM traffic class has higher priority and is switched by an optical packet switch while the lower priority best-effort SM traffic by an electrical one (or using electronic buffers). Therefore, the integrated system can include the parallel one, i.e. assign additional wavelengths only for SM as in PHON, to offer a bound on its QoS, depending on the available wavelength resources.

\section{SUB-WAVELENGTH AGGREGATION}

\section{A. Fusion Node}

Fusion is a technology building on the IHON architecture with the goal of enabling Ethernet packet transport for both types of traffic: emulating the wavelength service for GST with circuit QoS and increasing the wavelength utilization by adding SM packet traffic. Thus, the current implementation, the TransPacket H1 prototype node [21], moves from all-optical switching technologies e.g. optical packet switching, towards using standard Ethernet technology over the optical medium.

The block diagram of the hybrid node employed in this work is presented in Fig. 2(a). The node has two $10 \mathrm{~Gb} / \mathrm{s}$ Ethernet (10GE) interfaces which are connected to the wavelength transport channel. Ten $1 \mathrm{~Gb} / \mathrm{s}$ Ethernet (GE) interfaces are applied to increase the channel utilization by adding SM traffic or transport GST sub-wavelengths. Ethernet frames entering the hybrid network at a port of the Fusion node are tagged with a Virtual Local Area Network (VLAN) label indicating the end-to-end path and type of service, i.e. GST or SM. For enabling transparency to customers' traffic, forwarding on the Ethernet media access control (MAC) addresses is avoided. Hence, the VLAN tagging is applied similarly to the multiprotocol label switching (MPLS) approach. This enables the node to perform forwarding decisions based only on the port, the label and the associated service priority. Furthermore, the VLAN tag is removed at the edge destination node, enabling the transparency to the customer's traffic MAC addresses and VLANs in the hybrid domain. Both SM and GST follow end-to-end paths which can be manually provisioned through a management system or local node configuration. The key difference is that the GST is reserved absolute priority on its path while SM packets will be using the available leftover capacity.

All traffic entering the node has to be converted in the electronic domain to be processed. Locally, SM packets received at an input $10 \mathrm{GE}$ interface are either dropped to the GE port associated with its VLAN or queued at the output $10 \mathrm{GE}$ port waiting to be transmitted to the next node in the idle-time gaps. The GST packets received at the $10 \mathrm{GE}$ input interface pass through to the other 10GE output interface with absolute priority and light processing in the node, i.e. only the VLAN header, easing processing in any intermediate nodes.

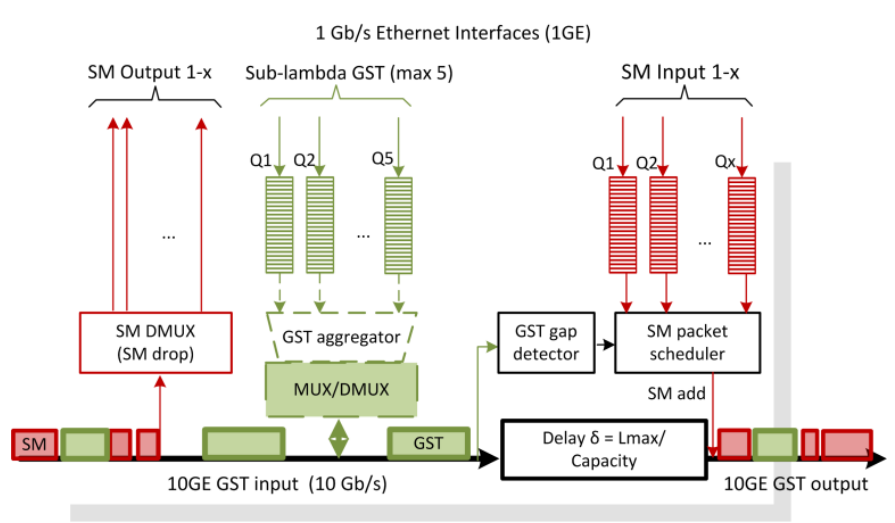

(a)

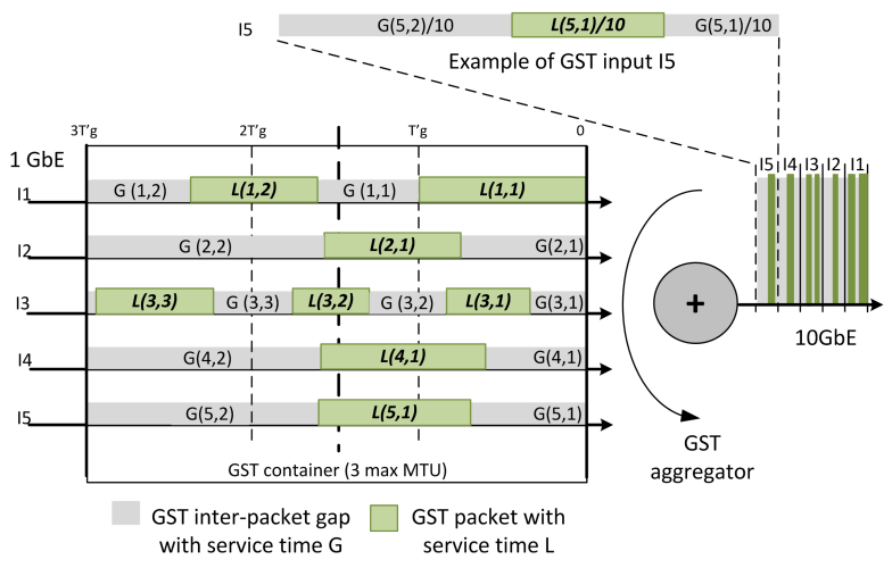

(b)

Fig. 2. (a) Fusion node block diagram. The example illustrates the left-to-right direction; (b) An example of the aggregation scheme of five GE GST input streams to one 10GE channel.

\section{B. Aggregation}

The key idea of the aggregation scheme is to transfer the information regarding the GST inter-packet gap in bytes when packets arrive at a port, i.e. GE port assigned to the GST stream. This information is then used by the aggregator when reading the packets from the buffer and transmitting them in the wavelength channel with the same number of bytes in between packets. Hence, the stream is sent out precisely as it arrived with the inter-packet gaps being preserved, theoretically enabling zero packet delay variation (PDV). Since the aggregation ratio is 1 to $10 \mathrm{~Gb} / \mathrm{s}$, the transmission time of a GST packet in the $1 \mathrm{GE}$ port is ten times slower than the transmission time at the $10 \mathrm{GE}$ port. Aggregation containers, e.g. a time period, reserved for each port will be calculated depending on the maximum expected GST packet size and number of ports that are 
being aggregated. Each of the containers is identified by a synchronization packet. Due to the added overhead, the principle allows scaling up to a maximum of nine ports.

Figure 2(b) illustrates the current implementation of the aggregation scheme employed in the field-trial. Five $1 \mathrm{~Gb} / \mathrm{s}$ Ethernet input streams are classified as GST, tagged with the corresponding VLAN tags when entering the GE ports, and aggregated into the $10 \mathrm{~Gb} / \mathrm{s}$ Ethernet output link. The latter is configured as a trunk port, i.e. it will transport all VLANs. Each of the inputs is logically divided into corresponding containers which begin with a synchronization packet. The duration corresponds to three 2048 Byte maximum transmission unit (MTU) 3T'g. The five packet sets are then aggregated into the $10 \mathrm{~Gb} / \mathrm{s}$ channel. Each sub-wavelength stream follows a reserved path. At the destination, the packets are extracted from their container and the packet gaps are used to precisely reconstruct the stream. Note that for the architecture in Fig. 2(a), the 10GE wavelength will be either configured for a single client $10 \mathrm{~Gb} / \mathrm{s}$ GST stream, or for aggregated client $1 \mathrm{~Gb} / \mathrm{s}$ GST streams. In both cases the additional GE ports will be used for adding/dropping SM traffic in the network.

\section{FIELD-TRIAL OF SUB-WAVELENGTH TRANSPORT}

\section{A. Setup}

The main goal of the experiment is to demonstrate subwavelength aggregation, transport and de-aggregation with circuit QoS: low deterministic delay, ultra-low packet delay variation and no packet loss. In addition, previously demonstrated IHON characteristics should be maintained, i.e. the GST circuit transport is independent of the insertion of the statistically multiplexed traffic that is applied for increasing the wavelength utilization efficiency [16]. In the carrier provider network of UNINETT, two Fusion nodes were connected through a fiber link of $3.25 \mathrm{~km}$ with a 10 $\mathrm{Gb} / \mathrm{s}$ Ethernet wavelength, as illustrated in Fig. 3. The fixed delay $\delta$ was set to $7.68 \mu \mathrm{s}$ to accommodate an SM MTU of 9600 Byte.

Five GST streams were added through the packet generator to the GE ports of $N_{1}$, aggregated into the $10 \mathrm{GE}$ wavelength channel, de-aggregated by $N_{2}$ to their corresponding ports and received for measurement back to the tester. The Ethernet packet length follows the empirical distribution in Table I, taken from Internet measurements [22]. The offered load $\rho_{g}$ of each GST stream $g$ is varied by changing the inter-packet length providing a total GST offered load in the wavelength $\rho_{G}=\sum_{g} \rho_{g}$. One SM stream was added from the tester to the second 10GE port of $N_{1}$ transmitted on the free time-gaps in the transport wavelength between the nodes, and sent back to the tester from $N_{2}$. The SM offered load $\rho_{s}$ was varied to demonstrate the increase in the achievable maximum throughput without any impact on the GST streams.

\section{B. Results}

The performance results of the GST aggregation scheme were gathered both through reference tests in the lab and in the field-trial. All the GST streams had an equal average load $\rho_{g}$ normalized over the $10 \mathrm{~Gb} / \mathrm{s}$ wavelength, offering a total GST load in the channel $\rho_{G}=5 \rho_{g}$. This load was varied from 0.01 to 0.5 with and without inserting SM traffic and both sets of results were consistent: the measured GST delay was found constant, independently of the GST or SM load in the system. The average GST delay of the end-to-end streams in the field-trial is $67.22 \mu \mathrm{s}$ where $16.22 \mu \mathrm{s}$ correspond to the propagation delay in the fiber link and 51 $\mu \mathrm{s}$ to the nodal delays. From the latter, $7.68 \mu \mathrm{s}$ corresponds to the fixed delay $\delta$ and $43.3 \mu$ s to the nodal processing delay $\left(N_{1}\right.$ and $N_{2}$ ). The average packet delay variation, i.e. delay variation between consecutive packets in a stream, is $15 \mathrm{~ns}$ with a measured maximum of $160 \mathrm{~ns}$. The minimum and maximum (boundary) delay values registered over all tests and loads differ with an average of $320 \mathrm{~ns}$, corresponding to the peak-to-peak PDV for GST.

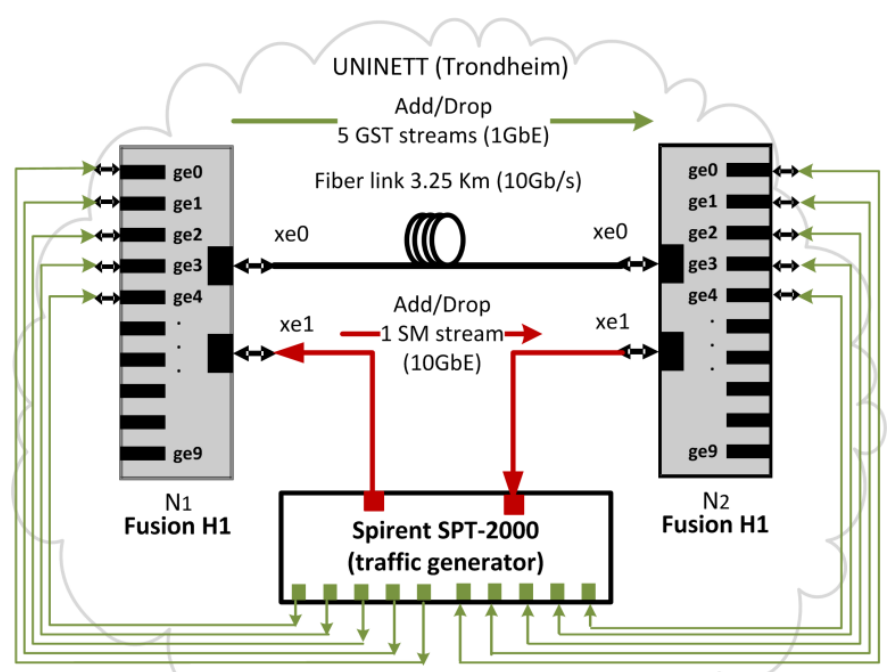

Spirent SPT-2000:

10 Ethernet $1 \mathrm{~Gb} / \mathrm{s}$ (GST streams)

2 Ethernet $10 \mathrm{~Gb} / \mathrm{s}$ (SM stream)
H1 Fusion nodes (N1, N2): 10 Ethernet $1 \mathrm{~Gb} / \mathrm{s}$ Interfaces (ge[0-9]) 2 Ethernet $10 \mathrm{~Gb} / \mathrm{s}$ Interfaces (xe[0-1])
Fig. 3. The field-trial setup with two prototype H1 nodes from TransPacket and Spirent SPT-2000 packet generator/tester. The GST connections are depicted in green while SM in red.

In Fig. 4 are presented the GST and SM end-to-end delay results in microseconds for two scenarios. In the first one, Fig. 4(a), the total offered load in the wavelength $\rho_{T}$ is equally offered by the GST aggregate and SM so that $\rho_{T}=\left(\rho_{G}+\rho_{S}\right)$ and $\rho_{G}=\rho_{S}$. The boundary SM delay values (min, max) can be seen plotted together with the average and illustrate how the increase in GST load influences the SM performance, i.e. the average delay and also the maximum delay bound rise. We observe that as both the GST and SM loads increase, the GST average delay is still

TABLE I

EMPIRICAL PACKET LENGTH DISTRIBUTION

\begin{tabular}{cc}
\hline \hline Packet Length & Probability \\
\hline 64 & 0.45 \\
594 & 0.1 \\
1318 & 0.05 \\
1418 & 0.05 \\
1518 & 0.35 \\
\hline \hline
\end{tabular}


constant. When $\rho_{T}$ reaches 0.746 with 0.375 GST load, the system goes into saturation: the SM traffic starts experiencing congestion and we observe SM packet losses, but the GST characteristics/QoS parameters do not change. We continue increasing the GST load at full capacity for each GST stream, i.e. $\rho_{G}=0.5$, without any impact on its performance, while the SM delay and packet loss continues to increase. The sub-wavelength granularity transport has absolute priority over SM and neither its delay, nor PDV nor loss are affected by the SM insertion.

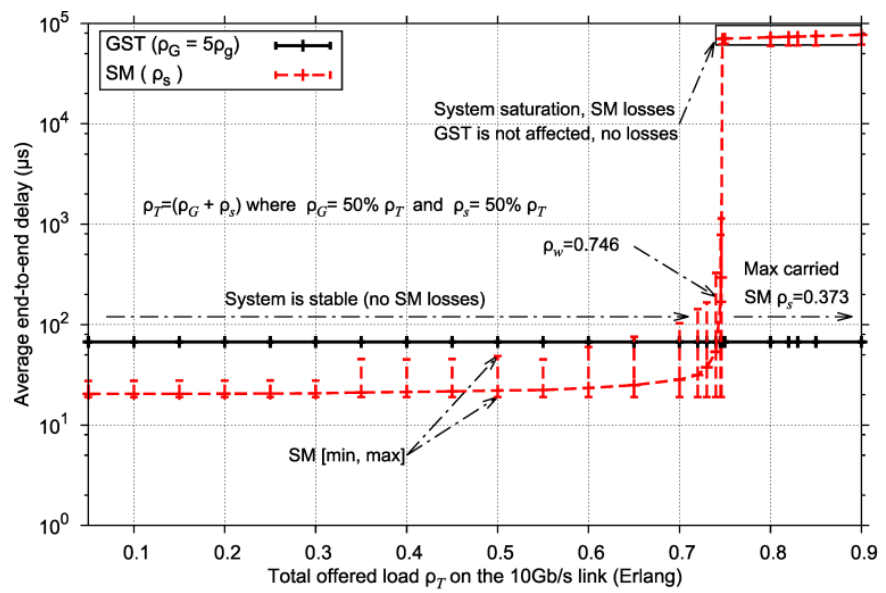

(a)

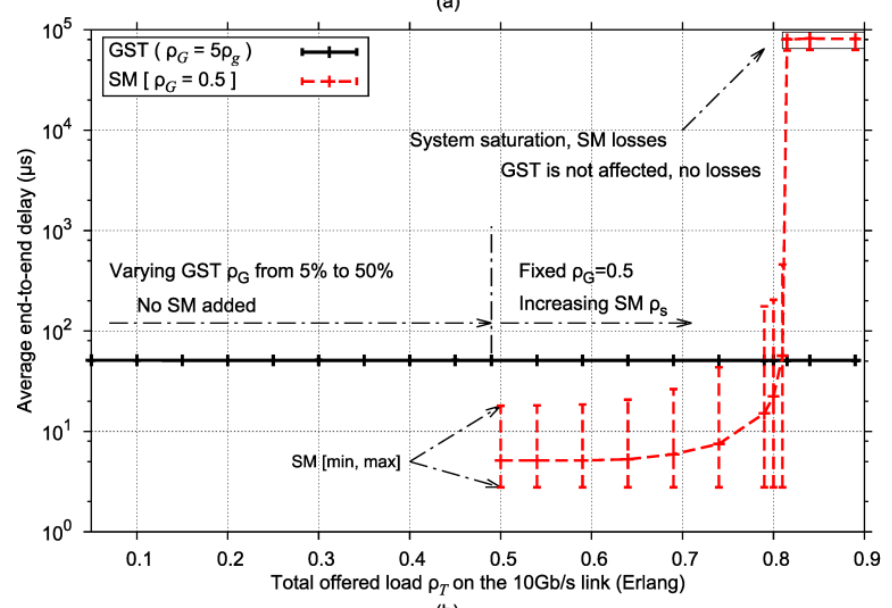

(b)

Fig. 4. Average end-to-end packet delay (in microseconds) as a function of the normalized offered load on the $10 \mathrm{~Gb} / \mathrm{s}$ Ethernet wavelength. (a) The total load in the channel is an equal contribution from both traffic classes; (b) the load in the channel up to 0.5 is offered only by GST and then additional SM traffic is added up to the saturation point.

In Fig. 4(b) is illustrated the performance of GST without any SM up to $\rho_{T}=\rho_{G}=0.5$, where additional SM traffic is added increasing $\rho_{T}$ until the SM saturation point is reached at $\rho_{T}=0.82$. In the stable system state, the SM delay in the first graph, Fig. 4(a) is higher, even though the GST load is smaller, i.e. equal to the SM load. The main contribution for this difference is the higher SM load in the first scenario, adding to the queuing delay. Nevertheless, note that in the field trial there is only one first-in first-out (FIFO) queue for SM at the output channel. Hence, high SM delays are mainly a result of the reservation of the channel for GST: long SM packets when reaching the head of the FIFO queue might not fit the gaps and will add a head-of-line blocking for shorter ones that might fit. When the system is in saturation, this is increasing the delay even further than the buffering time.

The experimental results show that the GST streams maintain their characteristics, i.e. inter-packet gap , during the aggregation and transport through the IHON network. Furthermore, results show the isolation of the GST streams from each other, i.e. no packet losses and the average delay remains constant (deterministic) regardless of the GST or SM load.

The maximum carried SM traffic was also evaluated in the test-bed. The offered GST load varied from $10 \%$ to $50 \%$ of the wavelength capacity with different combinations of the number $g$ of GST streams offering $\rho_{G}$. It was observed that the SM insertion increases the wavelength utilization with a maximum of $32.4 \%$ for $\rho_{G}=0.5$, up to $8.24 \mathrm{~Gb} / \mathrm{s}$ total throughput. Hence, the network shows to perform as a statistical multiplexing packet network with high utilization while still providing a service with circuit QoS properties.

\section{MaXimum Wavelength Utilization}

In the experimental test-bed the average packet length $\bar{L}$ of the streams was set to a five-modal empirical distribution as in Table I, taken from Internet measurement-based statistics, with emphasis on the 64B, 500B and 1500B packets. However, the setup was limited by the Spirent traffic generator, which creates the traffic load by fixing the inter-frame gap (IFG) so that the load for each stream $x$ is defined as $\rho_{x}=\bar{L} /(I F G+\bar{L})$. This traffic pattern is the worst case scenario for the SM insertion, as previously seen in other related works [16]. For example, for the same average GST load, a more bursty (random) GST traffic would allow for a more burstified (random) gap size thus increasing the probability of fitting the long SM packets at the head of the queue. Therefore, to generate different traffic patterns, the GST aggregation scheme was simulated through a discrete event simulator implemented in SIMULA programming language using the DEMOS library [23]. In Fig 5 are illustrated the results on the average SM nodal delay and packet loss probability for three cases:

1. Measured nodal delay in the experiment without the propagation and transmission delay components;

2. simulation with fixed inter-frame gap replicating the experiment to validate the simulator;

3. negative exponential distribution of the packet interarrival time.

In the latter, the SM packets are generated according to a Poisson arrival process. The GST traffic is generated by five on-off sources with exponentially distributed inter-arrival times (off-time) between packets. For both classes the packet length follows the same empirical distribution used in the experiment. The simulation results were obtained running ten independent replications to establish $95 \%$ confidence intervals. Delay statistics are collected for successfully scheduled SM packets when they exit the buffer. Each simulation run ends after having scheduled $10^{9}$ 
successful packets.

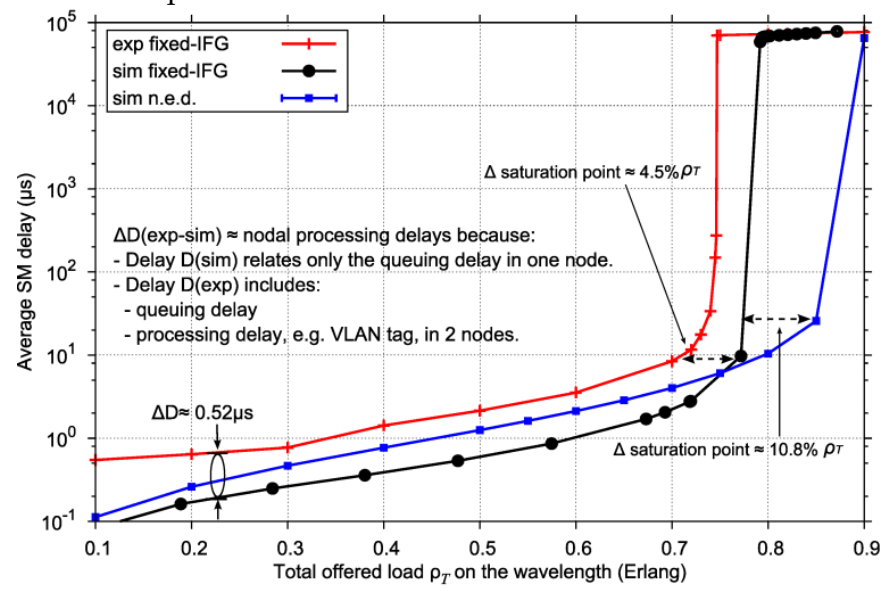

(a)

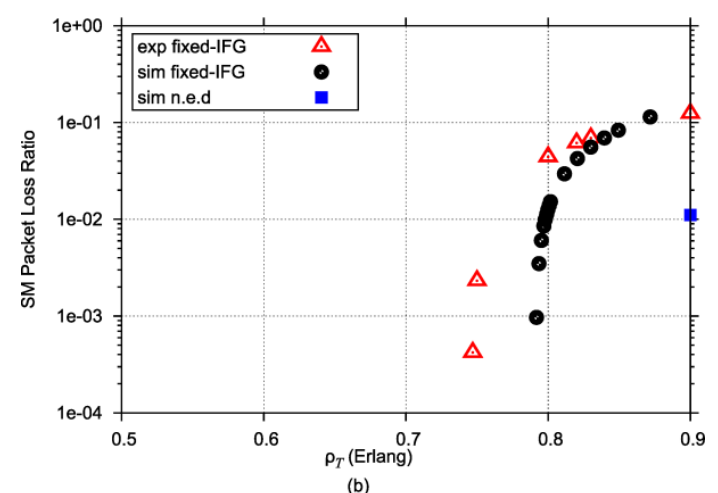

Fig. 5. (a) SM packet delay as a function of the normalized offered load on the wavelength. The simulation replicating the fixed IFG GST traffic pattern captures the queueing delay of SM at the first node due to contention with GST. The simulation results for n.e.d. of the GST inter-packet gaps illustrates that the actual saturation point is reached even further, e.g. at load 0.88/0.9. (b) SM packet loss ratio as a function of the GST aggregated load on the wavelength.

The simulation results on delay, obtained by replicating the traffic generation in the test-bed, capture the SM queuing delay at the insertion point in the channel and are close to the experimental results. The main differences in delay are the contribution of internal processing delay in the Fusion node. For example, the nodal processing delay includes serialization delays in the internal Fusion node buffering and the tagging process which is not included in the simulation of the system. The differences in the saturation point are related to the overhead within the physical Fusion implementation, i.e. timing information for synchronization of the containers. In case of Poisson arrivals for SM and on-off sources for GST, the results show that the saturation point is reached at a later load value allowing for an additional $10.8 \%$ channel utilization. The SM packet loss ratio is illustrated in Fig. 5(b). It is observed that as with the delay curve that rises steeply once the saturation point is reached, even in the simulator the first measurable packet losses are first observed only at the range of $10^{-4}$. The system uses deep $200 \mathrm{~ms}$ buffers to accommodate the SM load fluctuation. Nevertheless, the average SM delay before the saturation point is in the range of only microseconds, thus the main influence on the saturation point is not only the total SM load, but the GST load and the distribution of the gaps. This further obliges for a good knowledge on the bound of the maximum carried SM load that should be accepted/provisioned in the network as to provide a minimum QoS with acceptable packet loss probabilities for SM.

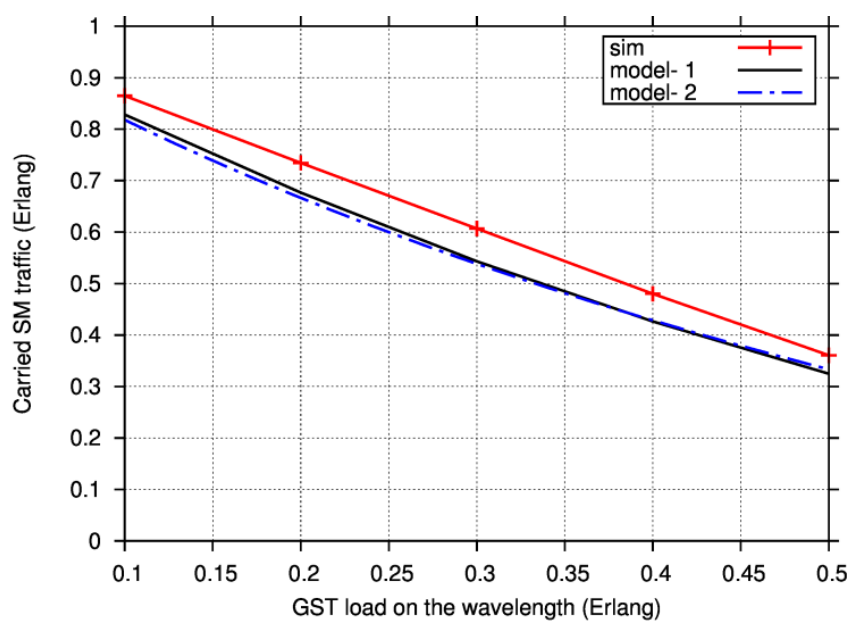

Fig. 6. The maximum carried SM (effective residual capacity) as a function for the GST load.

The simulator was then used to characterize the maximum achievable SM throughput in the system $\rho_{s}^{\max }$ as a function of the GST load $\rho_{G}$. The results of the carried SM load as a function of the GST load in the system are illustrated in Fig. 6. The simulation results were obtained by saturating the channel with SM packet traffic. In addition two analytical models quantifying the SM left-over capacity are provided to the network designer as a simple way of dimensioning the system. The first model as proposed in [16] finds the probability that the gap is wasted $\varepsilon$ equal to the probability that the inter-arrival times of GST packets are smaller than the mean service time of SM packets (assuming Poisson arrivals). This probability is used to quantify the leftover capacity which cannot be used, identified as wasted capacity. Hence, the effective left-over service available to $\mathrm{SM} \rho_{s}^{\max }$ is given by Eq. (1).

$$
\rho_{s}^{\max }=1-\rho_{G}(1+\varepsilon)
$$

The second model that can be applied is adapted from [15] where the system is saturated with SM. Hence it is assumed that an SM packet is always available in the buffer. To apply that model for the Fusion system under consideration, the SM real-time class load is set equal to 0 and only one wavelength channel is considered. The model finds first the probability of the successful transmission of an SM packet $\Pi_{s}$ defined as the frequency of the event 'successful transmission' over all events in the system, i.e.:

$$
\Pi_{s}=\frac{\mu_{s}}{\left(\lambda_{G}+\mu_{s}\right)}
$$

where $\mu_{s}$ is the SM service rate and $\lambda_{G}$ the arrival rate of GST packets allowed to interrupt the SM transmission. 
Note that the interrupted SM packets correspond to the wasted gaps of the previous model. The maximum carried SM load $\rho_{s}^{\max }$ would then be calculated simply by Eq. (3). Results from both models are also plotted in Fig. 6. The differences between the two models are very small, so that either model can be used for a safe bound on the maximum acceptable SM load for guaranteeing QoS to ensure the system does not saturate.

$$
\rho_{s}^{\max }=\Pi_{S}\left(1-\rho_{G}\right)
$$

\section{DISCUSSION}

Today's networks use a hierarchy of layered technologies requiring multiple protocol adaptions and encapsulations to carry IP traffic on the physical optical transport, e.g. IP/Ethernet over OTN over WDM. The benefit of the Fusion technology is that it removes additional layers needed for sub-wavelength services, offering Ethernet over WDM transport, thus highly reducing the complexity and the overhead. Comparing the integrated hybrid with a parallel hybrid solution, the results in Fig. 6 and $\rho_{s}^{\max }$ derived from the models, i.e. the additional carried SM traffic in a wavelength, can be expressed as the cost benefit of IHON versus PHON. Depending on the network connectivity, the available wavelength resources, and traffic demand matrix, the cost-efficiency of the architecture in terms of network throughput can be derived from $\rho_{s}^{\max }$.

\section{A. Fusion in the optical network}

The results are focused on demonstrating the aggregation scheme of sub-wavelengths, i.e. virtual wavelengths services, at the edge nodes of a point-to-point wavelength lightpath. The approach may be combined with optical cross-connect nodes with multiple wavelengths/fibers. A lightpath is then provisioned end-to-end and will be shared by fixed virtual-wavelength services. E.g. two Fusion nodes may be applied on the edges of the lightpath connection. The added SM traffic is then also transported all-optically, following the same lightpath through the network.

Furthermore, the virtual time slots switching may be extended from space to switching between wavelengths, and time. At core nodes switching between multiple inputs/outputs will be required. The highest buffer requirement occurs for the worst case scenario where a container number $i$ on an input wavelength has to be switched to the previous container number $i-1$ on an output wavelength; the container then has to be buffered till the appropriate container transmission time in the next frame. For the current scheme with five sub-wavelengths per physical wavelength the maximum added delay per node will be bound to $4 D$, where $D=3 T^{\prime} g$ is the transmission time of a container. If an all-optical solution for GST is desirable, it may be implemented with an FDL based buffer block per output wavelength. Each block with 4 FDLs of lengths multiples of $D$, enabling switching between all time slots. With the current FDL based technology for optical buffering, node size scalability issues arise; e.g. the length of the fibre per container will be approximately $1 \mathrm{~km}$.

The SM traffic is extracted on each hop as it is not necessarily following the GST path and processed electronically or alternatively using all Optical Packet Switching (OPS). The IHON architecture in [14] is an alternative for optical differentiation of SM and GST by using optical codes. If electronic header processing for differentiating the classes of service is applied, overhead for a guard band between the headers and payload for GST, as well as additional added delay for GST packet payload while the header is processed, may be introduced.

For extremely latency sensitive applications relying on short transmission delay and therefore transmission paths of less than approximately $10 \mathrm{~km}$ (50 Microseconds), the added fixed delay $\delta$ on each hop of the GST path may be an issue for the IHON systems. Different approaches can be taken for addressing the issue: (1) the 3LiHON approach [14] for real-time class, where no added delay is applied on time-sensitive traffic but pre-emption of the packets is allowed; (2) the Fusion added delay can be minimized to correspond to a maximum SM MTU of 1518 Byte equal to $1.2 \mu \mathrm{s}$. The jumbo SM frames would need to be segmented in the network. Nevertheless, the GST paths should be traffic engineered with the delay as a key constraint in the path selection

\section{B. The SM buffer size}

The results demonstrated in this work are related to the electronic implementation of the integrated hybrid optical network principle through the prototype Fusion technology. In this implementation all traffic is converted electronically on all nodes of the path. For future designs optical IHON architectures, that to a higher extent are applying optical processing, may be applied. The presented results relate to the Fusion system implementation with deep buffers for SM traffic, i.e. $256 \mathrm{MB}$, enabling ability to handle highly bursty GST traffic. Previous works have demonstrated the feasibility of an optical IHON implementation [18] with shallow SM buffers, where GST is transported all-optically. Furthermore, all-optical architectures have been proposed by applying fiber delay line (FDL) buffers for SM traffic [14], [19]. An all-optical solution should benefit from bit/protocol transparency and may have additional advantages of low energy consumption. However, the scheduling algorithm which is aware of the inter-frame gaps and the SM packets sizes available in the system, benefits from shallow electronic buffering as in [13] and [18] and may further benefit being applied together with the SM scheduling scheme described in Section II. This is especially beneficial for increasing the wavelength utilization when it is provisioned for timing sensitive GST traffic that cannot be burstified in the edge resulting in highly fragmented spare capacity for SM insertion. The drawback of the system is then the higher energy consumption as the SM share of traffic has to be converted to the electronic domain on each hop. Thus it is a trade-off between energy consumption versus network throughput and wavelength overprovisioning cost.

For the dimensioning of the SM buffer depth, the saturation point in the system is a key parameter. As results illustrated, once the system is in saturation the SM delay increases steeply and the deep buffers only add to the end-to-end delay of the SM packets successfully transported, 
but do not necessarily help with the PLR. The main reason is that the carried SM load is highly dependent on the GST load and the distribution of the gaps. Hence the system has to be first dimensioned for the maximum acceptable SM load as approximated in Eq. (1) and Eq. (3). Assuming that the system is in a stable condition the buffer depth requirement when moving to the optical domain can be less stringent and a buffer depth of tenths to hundreds of packets would suffice for an acceptable PLR. The SM delay curve in Fig. 4 (a) illustrates this. When the system is in stable condition before saturation, e.g. $\rho_{T}=0.77$, the average $\mathrm{SM}$ delay is $9.8 \mu \mathrm{s}$ resulting in an average of $16 \mathrm{SM}$ packets in the buffer. For smoothing microbursts in the core network with an acceptable SM PLR a buffer of hundreds of KB would be sufficient.

\section{CONCLUSION}

Integrated hybrid optical networks enable cost effective transport by combining packet switched throughput with circuit switched quality of service. In this work was demonstrated the first sub-wavelength transport on a wavelength through the integrated hybrid circuit/packet network, using Ethernet. Results confirm that the Fusion implementation of the IHON architecture with the additional sub-wavelength transport is able to: (1) maintain the stream characteristic with zero packet loss and ultralow packet delay variation, i.e. maintain its inter-packet timing through aggregation, transport and de-aggregation; (2) provide circuit-switching properties with a low deterministic delay and no packet loss; (3) increase the utilization of the wavelength through statistical multiplexing.

The high throughput efficiency was demonstrated by adding packet-switched statistical multiplexed traffic on the common circuit/packet lightpath. A total channel utilization of $82.4 \%$ was reached without packet loss on the SM class. For all loads, the GST sub-wavelengths remained unaffected for packet loss, delay and delay variation parameters.

The field-trial demonstrates the maturity of the integrated hybrid optical network technology and its capability of removing the timing obstacles for packet networks, matching the timing of OTN and legacy SDH/SONET networks. By employing IHON, packet networks are able to offer sub-wavelength services and are brought closer to fully replace legacy TDM-based networks. Comparing to parallel hybrid implementations that combine Ethernet packet switching and OTN circuit switching in the same node, IHON enables simplicity by using only Ethernet and increases the utilization of the wavelength resources in the network. It is especially relevant for multi-service edge/aggregation nodes in an optical domain serving Ethernet networks with wavelength and sub-wavelength services while maintaining its high bandwidth efficiency.

This paper demonstrates the feasability of the FUSION concept using technology currently available. For extending the Fusion concept to the optical domain, current optical circuit switching elements may be applied for switching the combined SM and GST signal. A further extension of IHON to the optical domain will be to apply all optical packet switching technology for switching the SM traffic, when this technology becomes more benefical than using electronics. Furthermore, for applications in local area networks handling extremely latency demanding applications requiring short-range transmission to keep the transmission delay low, the IHON may be extended to the $3 \mathrm{LiHON}$ architecture introducing a third ultra-low latency class of service.

\section{ACKNOWLEDGMENT}

This work was supported in part by the Norwegian Resource Council and by GN3plus JRA1-T1.

\section{REFERENCES}

[1] A. Saleh, and J.M. Simmons, "Technology and architecture to enable the explosive growth of the internet," IEEE Comm. Mag., vol. 49, no. 1, pp. 126-132, Jan. 2011.

[2] M. Xia, S. Dahlfort, L. Lu, G. Wang, and S. Shen, "CapEx Model and Analysis for Metro Networks: DWDM vs. Packet," in Proc. of Optical Fiber Communication Conference (OFC) 2014, paper W2A.12, March 2014,

[3] "Interfaces for the optical transport network (OTN)," ITU-T Recommendation G.709/Y.1331, 2009.

[4] M. Carroll, J. Roese, and T. Ohara, "The operator's view of OTN evolution," IEEE Comm. Mag., vol. 48, no. 9, pp. 46-52, 2010.

[5] A. Gumaste, and N. Krishnaswamy, "Proliferation of the optical transport network: A use case based study," IEEE Comm. Mag., vol. 48, no. 9, pp. 5461, 2010

[6] Z. Ghebretensae, J. Harmatos, and K. Gustafsson, "Mobile broadband backhaul network migration from TDM to carrier ethernet," IEEE Comm. Mag., vol. 48, no. 10, pp.102-109, 2010.

[7] S. Thiagarajan, M. Gemelos, and M. Ma, "Transport Network Evolution for Advanced Services," in Proc. Of Optical Fiber Communication Conference (OFC/NFOEC) 2013, paper NTh4J.4.

[8] A. Jukan, M. Veeraraghavan, and M.Z. Hasan, "Hybrid networking: evolution toward combined IP and dynamic circuit services [Guest Editorial]," IEEE Comm. Mag., vol. 49, no. 5, pp.112, May 2011

[9] A. Gumaste, and S. Akhtar, "Evolution of packet-optical integration in backbone and metropolitan high-speed networks: a standards perspective," IEEE Comm. Mag., vol. 51, no. 11, pp. 105-111, Nov. 2013.

[10] P. Iovanna, F. Testa, R. Sabella, A. Bianchi, M. Puleri, M. Casanova, and A. Germoni, "Packet-Optical Integration Nodes for Next Generation Transport Networks," J. of Opt. Commun. and Netw. (JOCN), vol. 4, no. 10, Oct. 2012.

[11] V. Eramo, M. Listanti, R. Sabella, and F. Testa. "Integrated OTN/WDM switching architecture equipped with the minimum number of OTN switches," J. of Opt. Commun. and Netw. (JOCN), vol. 6, no. 2, pp. 138-151, Feb. 2014.

[12] C.M. Gauger, J.P. Kuhn, E.V. Breusegem, M. Pickavet, and P. Demeester, "Hybrid optical network architectures: bringing packets and circuits together," IEEE Comm. Mag., vol 44, no. 8, pp. 36-42, 2006

[13] S. Bjornstad, D.R. Hjelme, and N. Stol, "A packet switched hybrid optical network with service guarantees," IEEE J. Sel. Areas Comm., vol. 24, no. 8, pp. 97-107, 2006.

[14] N. Stol, C. Raffaelli and M.Savi, "3-Level integrated hybrid optical network (3lihon) to meet future qos requirements," in Proc. Of IEEE GLOBECOM 2011, Houston, Texas, USA, Dec. 2011.

[15] W. Cerroni, and C. Raffaelli, "Analytical model of quality of service scheduling for optical aggregation in data centers," Photonic Network Communications, vol. 27 , no. 3, pp. 1-12, June 2014. 
[16] R. Veisllari, S. Bjornstad, and K. Bozorgebrahimi, "Integrated Packet/Circuit Hybrid Network Field-Trial with Production Traffic," J. of Opt. Commun. and Netw. (JOCN), vol. 5, no 10, Oct. 2013.

[17] S. Bjornstad, R. Veisllari, J. P. Braute, and K. Bozorgebrahimi, "Integrated Packet/Circuit Hybrid Network Field-Trial Demonstrating Sub-Wavelength Aggregation", in Proc. of Optical Fiber Communication Conference (OFC) 2014, OSA Technical Digest (online), paper W4H1.4, San Francisco, 2014.

[18] S. Bjornstad, M. Nord, V. L. Tuft, O. Austad, D. R. Hjelme, and L. E. Eriksen, "Experimental Demonstration of OpMiGua Hybrid Circuit/Packet Nodes," in Proc. of European Conference on Optical Communications (ECOC), 2006.

[19] A. Kimsas, S. Bjornstad, H. Overby, and N. Stol, "Reservation techniques in an OpMiGua node," in Proc. of Optical Network Design and Modeling, pp. 146-155, Springer Berlin Heidelberg, 2007.

[20] H. Harai, "Optical Packet \& Circuit Integrated Network for Future Networks (Invited)," IEICE Trans. Comm., vol. E95-B, no. 3, pp. 714-722, March 2012.

[21] TransPacket whitepaper, "TRANSPACKET H1; a fusion networking add-drop muxponder," [Online], Available: http://www.transpacket.com/fusionh1/fusion-ethernet-h1/, last checked June 2014.

[22] CAIDA, "Traffic Analysis Research", [Online]. Available: http://www.caida.org/research/traffic-analysis/pkt_size distribution/, last checked June 2014.

[23] G.M. Birtwistle, DEMOS-a system for discrete event modelling on Simula. University of Sheffield, England, 2003. 